Gut, 1978, 19, 783-786

\title{
Gastric emptying after treatment of stenosis secondary to duodenal ulceration by proximal gastric vagotomy and duodenoplasty or pyloric dilatation
}

\author{
C. M. White, L. K. HARDING, M. R. B. KEIGHLEY, N. J. DORRICOTT, AND \\ J. ALEXANDER-WILLIAMS
}

From the General Hospital and Dudley Road Hospital, Birmingham

SUMMARY A consecutive series of 12 patients with stenosis secondary to duodenal ulceration were treated by proximal gastric vagotomy (PGV) and duodenoplasty or PGV and dilatation of the stenosis. Three months after operation the rate and pattern of gastric emptying of a solid meal was measured in each patient and compared with 18 patients with uncomplicated duodenal ulcer treated by PGV alone. Two patients developed gastric stasis in the early postoperative period which resolved with medical treatment. All patients were asymptomatic and were eating normally three months after operation. There was no significant difference in the rate of gastric emptying postoperatively between the patients who had stenosis and those who had uncomplicated duodenal ulcers. These results indicate that despite early postoperative difficulties in some patients pyloric dilatation or duodenoplasty with PGV are both effective treatments for stenosis due to duodenal ulceration.

The superior early results of proximal gastric vagotomy (PGV) compared with other operations for duodenal ulcer are due to the preservation of an intact and innervated pyloric antrum (Johnston and Wilkinson, 1970; Clarke and Williams, 1972; Amdrup et al., 1974). However, gastric outlet obstruction is usually considered a contraindication to PGV unless it is combined with a drainage procedure. Pyloric or duodenal stenosis due to duodenal ulceration is usually treated by procedures that destroy the pylorus or bypass the pyloric antrum and are therefore likely to produce dumping, diarrhoea and bile vomiting (Wastell, 1969; Goligher, 1970).

Johnston suggested that patients with duodenal ulcer complicated by stenosis should be treated by PGV and dilatation of the stenosis (Johnston et al., 1973). He also reported that the symptomatic results in patients with pyloric stenosis were better after PGV and dilatation than after truncal vagotomy and drainage (McMahon et al., 1976). Duodenoplasty is an alternative method of treating the stenosis without destroying the pylorus. The symptomatic results of PGV and duodenoplasty in 25 patients with pyloric stenosis were indistinguishable from those in patients with uncomplicated ulcer treated by PGV alone

Received for publication 7 March 1978
(Kennedy, 1976).

Dilatation of the stenosis may be complicated by re-stenosis and in Johnston's series of 23 patients, two required reoperation for gastric stasis, whereas none of Kennedy's patients developed stasis after PGV and duodenoplasty. In both of these series only clinically apparent gastric stasis was recorded and no objective measurements of gastric emptying were made. Gastric stasis is often difficult to detect clinically (Roth et al., 1962; Hildebrand and Thomson, 1964) and the incidence of stasis may have been higher than these reports suggest.

The effects of a narrowed gastric outlet depend on the balance between the resistance to outflow and the propulsive activity of the antrum. A dynamic study of gastric emptying is the best way of assessing these effects. This paper reports on the rate and pattern of gastric emptying in patients with duodenal or pyloric stenosis treated by PGV and dilatation or duodenoplasty compared with patients with uncomplicated duodenal ulcer treated by PGV alone.

\section{Methods}

PATIENTS

A consecutive series of 12 patients with duodenal ulcer stenosis under the care of three surgical firms at 
the General Hospital, Birmingham, between November 1976 and August 1977, were treated by PGV and duodenoplasty (five patients) or PGV and dilatation (seven patients). For comparison we have also studied 18 patients with uncomplicated duodenal ulcer treated by PGV alone. The mean age of the patients with stenosis was 54.4 years (range: 38-68 years) and three were female. The mean age of the group with uncomplicated ulcer was 44.4 years (range 26-63 years) and two were female.

Multiple criteria have been used to select patients with significant gastric outlet obstruction because pyloric or duodenal stenosis presents with different symptoms (Balint and Spence, 1959). A modification of the criteria used by Balint and Spence has been used. The criteria were divided into major and minor groups and patients were included in the study only if they manifested at least (1) two major or (2) one major and two minor criteria. The clinical details of the 12 patients with stenosis are shown in the Table.

Table Criteria for diagnosis of duodenal ulcer stenosis (after Balint and Spence (1959)) and details of clinical features of patients in this study

Number of patients $(n=12)$

Major criteria

1. Classical history: persistent vomiting of

large amounts at times when the stomach

should be empty, usually containing

recognisable residues of food eaten some

hours previously

2. Visible gastric peristalsis

3. Mean aspirates, during hourly nasogastric

aspiration, totalling more than $1000 \mathrm{ml} / 24 \mathrm{~h}$

4. Retention of barium: stomach emptying

by less than two-thirds in $4 \mathrm{~h}$

11
1
6
6
1
8
1
5
12

OPERATION

The patients with stenosis were treated by PGV and duodenoplasty whenever possible using the technique of Kennedy (1976), with a two layer closure using chromic catgut and silk sutures. If the surgeon judged it impossible to enlarge the stenosis adequately without damaging the pyloric ring PGV and dilatation was performed. Our technique of pyloric dilatation differs from that described by Johnston and his colleagues (McMahon et al., 1976). After mobilising the duodenum stay sutures were placed distal to the stenosis and a $2 \mathrm{~cm}$ longitudinal duodenotomy was made. The stenosis was gently dilated with graduated Hegar dilators inserted through the duodenotomy (Fig. 1). The stenosis usually dilated readily to $14 \mathrm{~F}$ gauge but the dilatation achieved ranged from 12 to $17 \mathrm{~F}$. The duodenotomy was then closed transversely in two layers with chromic catgut and silk sutures.

Operations were performed by six surgeons from three surgical firms.

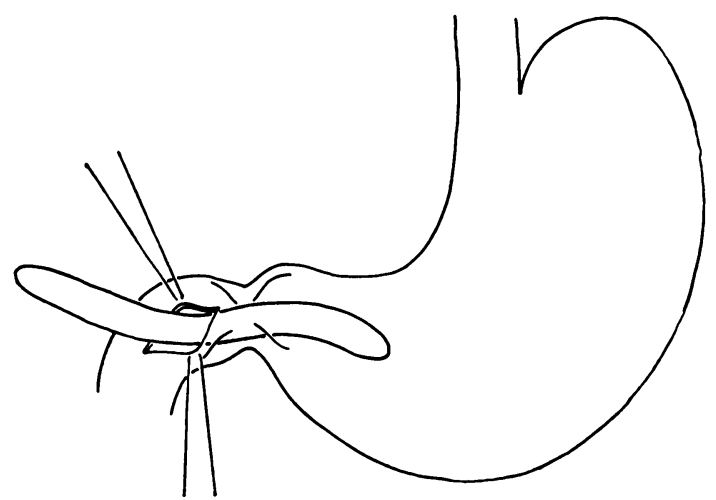

Fig. 1 Dilatation of the stenosis through a duodenotomy

GASTRIC EMPTYING

Three months after operation the rate and pattern of gastric emptying of a test meal was studied in all the patients. The meal was made from $100 \mathrm{~g}$ of tinned minced beef, $15 \mathrm{~g}$ of dried peas and $25 \mathrm{~g}$ of proprietary potato powder. A glass of milk $(150 \mathrm{ml})$ was taken with the meal. The three components of the meal and the milk were labelled during preparation with 113 Indium DTPA $(100 \mu \mathrm{Cl})$. The total volume was $400 \mathrm{ml}$.

An external scanning technique that allows the rate and pattern of emptying to be recorded from the start of the meal was used (Donovan, 1976a). Throughout the test the patient was seated erect. The initial passive emptying phase (Donovan, 1967a) was measured by calculating the maximum volume of meal recorded in the stomach (peak volume). The peak volume is low if rapid initial emptying has taken place during ingestion of the meal. If the initial emptying is slow the peak volume will be higher. The remainder of the meal, usually the majority, is emptied in approximately exponential fashion. The half emptying time ( $\left.\mathrm{T}_{2}\right)$, measured from the peak volume, was therefore chosen as the best measure of the rate of this phase of emptying (Fig. 2).

Student's $t$ test and Wilcoxon's rank sum test have been used to examine the statistical significance of the results. 


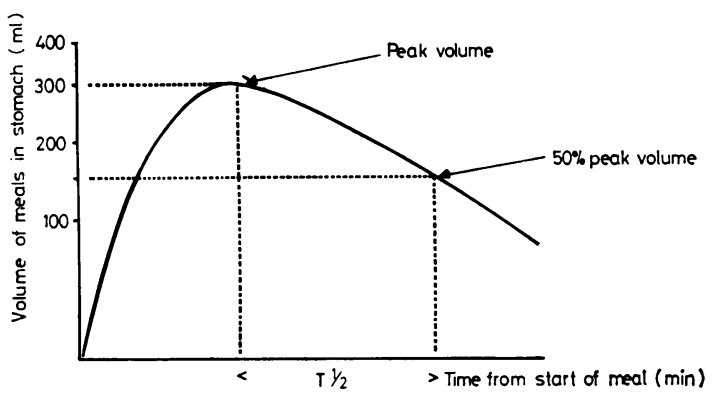

Fig. 2 A semilogarithmic plot of volume of meal in stomach versus time.

\section{Results}

\section{GASTRIC EMPTYING}

The $\mathrm{T}_{\frac{1}{2}}$ values are shown in Fig. 3. In the group with stenosis the $T \frac{1}{2} s$ ranged from 30.2 minutes to 122 minutes. In the uncomplicated ulcer group the values ranged from 24 minutes to 150 minutes. The distribution of results in the group with uncomplicated ulcer is not normal. It is either skewed or represents two separate populations. After logarithmic transformation Student's $t$ test and Wilcoxon's rank sum test have both been applied to the results and no significant difference in $T \frac{1}{2} s$ was found between the two groups. If the patients with stenosis are considered separately there is no apparent difference in the

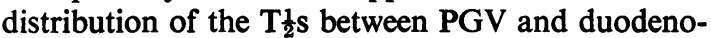
plasty and PGV and dilatation.

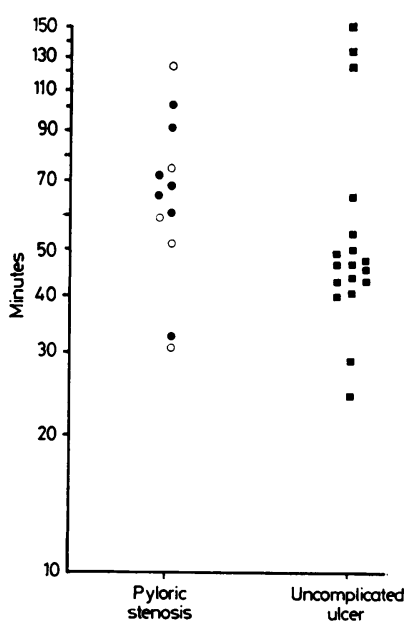

Fig. 3 The distribution of half emptying time ( $\left.T_{\frac{1}{2}}\right)$ three months after operation (logarithmic scale) PGV and dilatation . PGV and duodenoplasty $\bigcirc . P G V$ alone $\square$.
The peak volumes are shown in Fig. 4. The distribution is normal in both groups. The mean peak volume in the group with stenosis was $315 \pm 34.3 \mathrm{ml}$ SD and in the uncomplicated ulcer group was $327 \pm$ $37.5 \mathrm{ml} \mathrm{SD}$. These differences are not statistically significant.

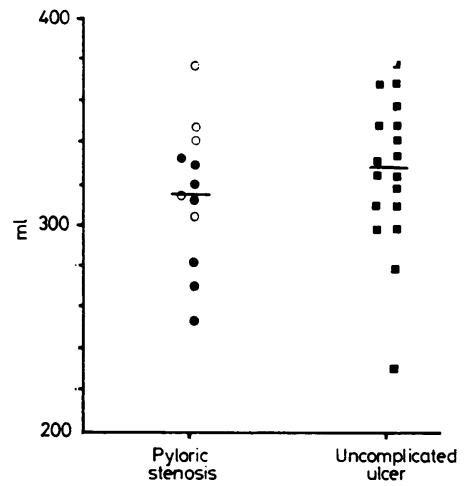

Fig. 4 The distribution of peak volume three months after operation. The mean is shown by a bar PGV and dilatation O. PGV and duodenoplasty $\bigcirc$. PGV alone $\square$.

\section{CLINICAL RESULTS}

None of the patients underwent reoperation for gastric stasis. One patient, whose stenosis was treated by PGV and dilatation started vomiting immediately after the operation. He was treated by intermittent nasogastric aspiration, intravenous replacement of fluids and electrolytes, and metoclopramide. After eight days his gastric aspirates were decreased to normal volumes and the nasogastric tube was removed. He was discharged home taking metoclopramide orally but continued to vomit occasionally for two months. The patient was adamant that he did not want a further operation. A second patient, also treated by PGV and dilatation, required nasogastric aspiration for six days after operation but was asymptomatic after the nasogastric tube was removed. Despite these early difficulties by three months all the patients were asymptomatic and eating normal meals.

\section{Discussion}

No significant difference in gastric emptying of the test meal has been demonstrated between patients with duodenal ulcer stenosis treated by PGV and duodenoplasty or PGV and dilatation compared with patients with uncomplicated ulcer treated by PGV alone. These results indicate that PGV and duodenoplasty and PGV and dilatation are both 
effective methods of treating duodenal ulcer stenosis.

The range of $T_{2}^{\frac{1}{2}}$ in normal individuals for this method is 30-70 minutes (Donovan, 1976b). Three patients after PGV alone had a prolonged $\mathrm{T}_{2}^{1}$; this may have been due to minor stenosis which was unrecognised preoperatively or alternatively, and more likely, the antral innervation may have been damaged during PGV. Three patients in the group with stenosis had slow emptying with $\mathrm{T}_{2}^{1}$ values of 122 minutes (PGV and duodenoplasty), 102 minutes (PGV and dilatation), and 89 minutes (PGV and dilatation). These results may either be due to operative damage to the antral innervation or due to restenosis. Evidence exists which suggests that if the delay in emptying is due to antral denervation it will usually improve with time (Cowley et al., 1972; Donovan et al., 1974). If re-stenosis is the cause of delayed emptying it may worsen. The gastric emptying tests will be repeated in our patients one year after operation.

A theoretical advantage of duodenoplasty is that re-stenosis might be less likely than after dilatation. Contrary to Kennedy's reported experience, duodenoplasty was possible in less than half of our patients (five of 12). However, our results suggest that there are no advantages to be gained by performing a duodenoplasty rather than dilatation. Neither of the two patients who had gastric stasis after PGV and dilatation had prolonged $\mathrm{T}_{2} \frac{1}{2}$ values when tested after three months. It is often difficult to make an adequate duodenoplasty in a scarred, oedematous duodenum. If the incision is too small gastric stasis may not be cured. This may explain the prolonged $\mathrm{T} \frac{1}{2}$ of 126 minutes seen in one patient. If the incision is enlarged to ensure a patent lumen the pylorus may be damaged. Dilatation of the stenosis may therefore be preferable, especially if duodenoplasty is technically difficult.

The technique of dilatation of the pylorus through a duodenotomy which we have used was chosen because it was less likely to damage the antrum and its nerve supply than gastrotomy. On the other hand a duodenotomy may be more likely to leak than a gastrotomy (McMahon et al., 1976). It is possible that in many cases even dilatation of the stenosis is unnecessary, PGV alone could be adequate. A stenosis due to fibrotic scar tissue is rare; the narrowing is usually due to a combination of the underlying fibrous contracture and the inflammatory oedema surrounding the ulcer (Bockus, 1943). PGV alone would heal the ulcer and, by allowing the oedema to subside, would relieve the obstruction. Unless a recurrent ulcer developed further stenosis would not occur.
Our early results suggest that PGV and duodenoplasty and PGV and dilatation are effective in the treatment of duodenal ulcer stenosis. They offer less risk of postoperative dumping, diarrhoea, and bile vomiting than do truncal vagotomy and drainage or gastrectomy.

\section{References}

Amdrup, E., Jensen, H. E., Johnston, D., Walker, B. E. and Goligher, J. C. (1974) Clinical results of parietal cell vagotomy (highly selective vagotomy) two to four years after operation. Annals of Surgery, 180, 279-284.

Balint, J. A., and Spence, M. P. (1959). Pyloric stenosis. British Medical Journal, 2, 890-894.

Bockus, H. L. (1943). Gastroenterology, vol. 1, p. 602. Saunders: London.

Clarke, R. J., and Williams, J. A. (1972). The effect of antral denervation and pyloroplasty on gastric emptying and 'dumping'. (Abstract) British Journal of Surgery, 59, 903.

Cowley, D. J., Vernon, P., Jones, T., Glass, H. I., and Cox, C. G. (1972). Gastric emptying of solid meals after truncal vagotomy and pyloroplasty in human subjects. Gut, 13, 176-181.

Donovan, I. A. (1976a). The different components of gastric emptying after gastric surgery. Annals of the Royal College of Surgeons of England, 58, 368-373.

Donovan, I. A. (1976b). Gastric Emptying. Thesis: University of Birmingham.

Donovan, I. A., Clarke, R. J., Gunn, I. F., and AlexanderWilliams, J. (1974). A comparison of gastric emptying at 3 and 12 months after proximal gastric or selective vagotomy without pyloroplasty. British Journal of Surgery, 61, 889-892.

Goligher, J. C. (1970). The comparative results of different operations in the elective treatment of duodenal ulcer. British Journal of Surgery, 57, 780-783.

Hildebrand, H., and Thomson, F. B. (1964). Stasis gastric ulcer: a complication of duodenal ulcer. Canadian Medical Association Journal, 90, 915-919.

Johnston, D., Lyndon, P. J., Smith, R. B., and Humphrey, C. S. (1973). Highly selective vagotomy without a drainage procedure in the treatment of haemorrhage, perforation, and pyloric stenosis due to peptic ulcer. British Journal of Surgery, 60, 790-797.

Johnston, D., and Wilkinson, A. R. (1970). Highly selective vagotomy without a drainage procedure in the treatment of duodenal ulcer. British Journal of Surgery, 57, 289-296.

Kennedy, T. (1976). Duodenoplasty with proximal gastric vagotomy. Annals of Royal College of Surgeons of England, 58, 144-146.

McMahon, M. J., Greenall, M. J., Johnston, D., and Goligher, J. C. (1976). Highly selective vagotomy plus dilatation of the stenosis compared with truncal vagotomy and drainage in the treatment of pyloric stenosis secondary to duodenal ulceration. Gut, 17, 471-476.

Roth, J. L. A., Vilardell, F., and Affolter, H. (1962). Post vagotomy and gastric stasis. Annals of the New York Academy of Sciences, 99, 203-212.

Wastell, C. (1969). Long-term clinical and metabolic effects of vagotomy with either gastrojejunostomy or pyloroplasty. Annals of the Royal College of Surgeons of England, 45, 193-211. 